\title{
ESTUDO DA BIODISPONIBILIDADE DE METAIS NOS SEDIMENTOS DA LAGOA DA PAMPULHA
}

Claudia de Vilhena Schayer Sabino*, Geraldo Frederico Kastner e Angela Maria Amaral

Centro de Desenvolvimento da Tecnologia Nuclear, Comissão Nacional de Energia Nuclear, CP 941, 30123-970 Belo Horizonte - MG

Simone Cristina Alves Lobo Leite, Ricardo Pinto de Carvalho e Isabel Reis e Silva

Pontifícia Universidade Católica, Av. Dom José Gaspar, 500, 30535-610 Belo Horizonte - MG

Recebido em 18/3/03; aceito em 29/9/03

\begin{abstract}
STUDY OF THE BIOAVAILABILITY OF METALS IN THE SEDIMENTS OF LAGOA DA PAMPULHA (PAMPULHA LAKE). The presence of metals in the sediments of Pampulha Lake was investigated in order to determine the bioavailability of metals in these sediments for the cultivation of vegetables such as lettuce. The chemical analyses of metals and As were accomplished by the $\mathrm{k}_{0}$ neutron activation technique. The plans for recovering this lake foresee the removal of the deposited sediments and transport of the discarded material to a specific area after the dam. The present study suggests that this strategy of discarding sediments doesn't imply any risk for the local population, in relation to the bioavailability of metals in these sediments.
\end{abstract}

Keywords: bioavailability; neutron activation analysis; lake sediment.

\section{INTRODUÇÃO}

A bacia hidrográfica da Pampulha, situada em Belo Horizonte, MG, é parte do rio das Velhas, sendo a superfície a montante da represa aproximadamente de $97 \mathrm{~km}^{2}$.

A partir do início dos anos 70, a lagoa vem sofrendo processo de assoreamento e poluição de suas águas e sedimentos, com o incremento da ocupação desordenada da bacia hidrográfica a montante. Isto tem trazido inúmeros transtornos à população, como a proliferação de insetos na região, o impedimento da utilização adequada da represa como área de lazer, a ocorrência de enchentes e a perda da identidade do complexo da lagoa.

Com a descaracterização, o volume de água, segundo estudos batimétricos realizados pelo CDTN/CNEN em 1999, era de 8.480.000 $\mathrm{m}^{3}$, isto é, $50 \%$ de seu volume original $\left(18.000 .000 \mathrm{~m}^{3}\right)$, o espelho d'água encontra-se reduzido a apenas $66,7 \%$ do seu valor inicial ${ }^{2}$.

O poder público vem atuando no sentido de recuperar o local para as finalidades para as quais foi construído: área de lazer e, também, amortecimento de cheias na bacia. Esta recuperação está sendo feita através do projeto PROPAM (Programa de Recuperação e Desenvolvimento Ambiental da Lagoa da Pampulha) com o saneamento das águas afluentes.

Com a retirada progressiva dos sedimentos, a capacidade do reservatório é aumentada, melhorando a laminação e o amortecimento das cheias. A tendência futura na bacia hidrográfica é de ocorrência de cheias maiores, considerando a mesma precipitação pluviométrica, em função do aumento da impermeabilização de sua superfície, pela ocupação urbana ${ }^{3}$.

O desassoreamento da Lagoa da Pampulha tem, como alternativa mais viável, sob o ponto de vista técnico e econômico, a remoção dos sedimentos ali depositados, através da utilização de draga de sucção e recalque, e a adução deste material, através de tubulação, para áreas de bota-fora a jusante da represa (Dragagem de Longa Distância - DLD), sendo aproveitado o grande desnível $(125$ m/17,8 $\mathrm{km}$ ) entre o espelho d'água e a confluência com o Rio das Velhas ${ }^{3,4}$.

*e-mail: sabinoc@cdtn.br
Neste processo, a fração arenosa poderá ser reaproveitada para utilização em construção civil (aterros, material de base para ruas e capas asfálticas e outros). A silto-argilosa (cerca de 70\%) deverá ser preferencialmente depositada em áreas de bota-fora, aterros sanitários, ou parte da mesma reintegrada aos cursos d'água, a jusante. Nestes locais, o material poderá ser utilizado pela população para plantio de alimentos, como as hortaliças. O conhecimento da biodisponibilidade de metais nestes sedimentos é importante, uma vez que, quantidades inadequadas podem ser absorvidas pelas plantas ${ }^{5}$, prejudicando o seu desenvolvimento ou causando danos às populações que delas se utilizarem.

Vários autores afirmam que espécies de vegetais e até cultivares diferem quanto à sua capacidade de absorção e acúmulo de metais. A alface foi escolhida para esse estudo por ser considerada uma das hortícolas mais eficientes na absorção de tais elementos ${ }^{6}$, além de seu fácil cultivo e manejo e um rápido período de tempo entre o plantio e a colheita.

A espécie escolhida foi a Alface Babá de Verão Manteiga (Lettuce sativa $L$.) por se tratar de uma variedade vigorosa, que apresenta folhas grandes, lisas e tenras ${ }^{7}$.

Este trabalho teve como objetivo o estudo da biodisponibilidade de As e alguns metais: Al, Ba, Ce, Co, Cr, Fe, K, La, Mn, Na, Rb, Sc, e $\mathrm{Zn}$, nestes sedimentos. O desenvolvimento dos vegetais foi estudado paralelamente, através de medidas lineares, com o objetivo de verificar como o substrato interfere neste processo.

\section{PARTE EXPERIMENTAL}

Um esquema mostrando as diferentes etapas do estudo está apresentado na Figura 1.

\section{Coleta de sedimentos}

A coleta foi feita próxima à Ilha dos Amores, onde existe uma diminuição na vazão e conseqüente deposição dos sedimentos.

As amostras de sedimento em suspensão foram colhidas em recipientes especialmente fabricados, de polietileno de alta densidade molecular, a fim de minimizar a possível adsorção dos metais nas paredes dos mesmos. 


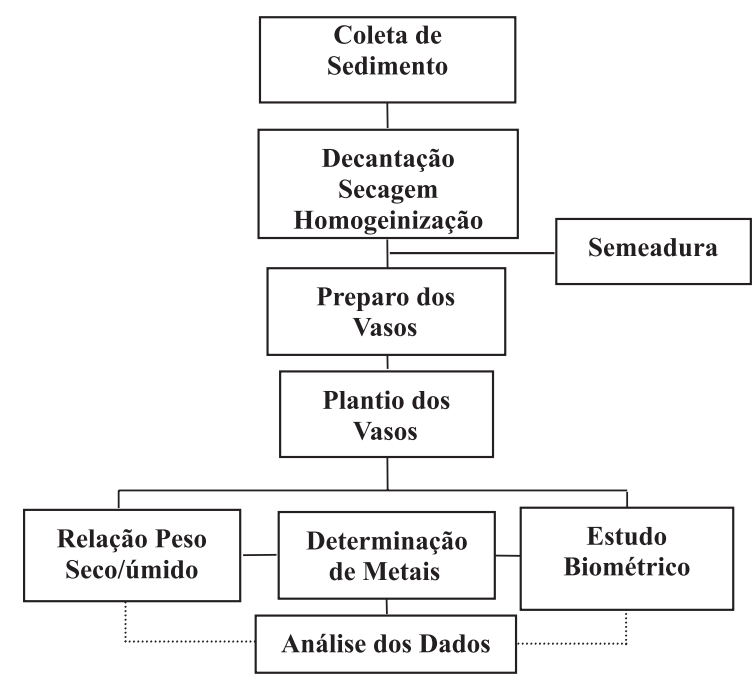

Figura 1. Esquema da metodologia

Após um período de 24 h, necessário para a decantação, a água foi descartada e o sedimento disposto em bandejas apropriadas para a secagem, à temperatura ambiente. O material foi então homogeneizado e foram retiradas um mínimo de 5 alíquotas para análise e cultivo.

\section{Cultivo da alface}

Para a semeadura, foram utilizadas bandejas de isopor e o número de mudas foi maior que o necessário para os vasos, pois, para o transplante foram escolhidas as melhores mudas, isto é, as mais saudáveis, com maior altura e número de folhas. O tempo esperado entre a semeadura e o transplante foi de aproximadamente um mês.

Para o transplante das mudas foram utilizados vasos de polietileno. O substrato foi preparado de três formas: $100 \%$ de terra vegetal (10 vasos); $50 \%$ de terra vegetal e $50 \%$ de sedimento (10 vasos) e $100 \%$ de sedimento (10 vasos). O transplante foi realizado no período mais fresco do dia.

Durante o crescimento, diariamente, após a rega foram feitas análises do desenvolvimento das plantas utilizando a medida de dimensões lineares.

A colheita foi realizada no momento de desenvolvimento máximo da planta, ou seja, quando apresentaram cabeças firmes e folhas tenras sem sinal de florescimento. Isso ocorreu 75 a 90 dias após o semeio. As plantas coletadas foram lavadas e colocadas em saco plástico etiquetado e então destinadas ao laboratório.

\section{Análises}

As amostras de alface foram pesadas e levadas à estufa, em temperatura próxima a $100{ }^{\circ} \mathrm{C}$ durante $48 \mathrm{~h}$. Após este período, foram pesadas e o fator de umidade (FCU) calculado. As amostras de sedimento foram secas a uma temperatura de $70{ }^{\circ} \mathrm{C}$ durante um período de $72 \mathrm{~h}$ e pesadas.

As análises foram executadas pela técnica paramétrica de ativação neutrônica $\mathrm{k}_{0}^{8} \mathrm{e}$ as irradiações foram feitas no reator TRIGA IPR-R1. As amostras e os padrões, com massa de aproximadamente $0,3 \mathrm{~g}$, foram acondicionadas em tubos de polietileno, próprios para a irradiação e, a seguir, colocados em tubos de poliestireno ("coelhos"), que foram irradiados por 5 min para isótopos de meia vida curta e por $16 \mathrm{~h}$ para determinação de isótopos de meia vida média e longa. Simultaneamente foram irradiados materiais de referência. Após a irradiação, amostras e referências foram contadas, uma a uma, em sistema de espectrometria gama constituído de detector HPGe de eficiência relativa de $50 \%$ e resolução de $1,8 \mathrm{keV}$ em $1332,2 \mathrm{keV}$; eletrônica processadora de sinal (DAS-2000 - CANBERRA); sistema de multicanal Genie-2000 e analisador de espectros.

O controle da qualidade foi feito através da utilização de replicatas de algumas amostras e da análise de materiais de referência: Soil-7, Polluted Farmland Soil e Mixed diet, certificados pela Agência Internacional de Energia Atômica, e GXR-6 da "United States Geological Survey".

\section{RESULTADOS}

As seguintes medidas relativas ao acompanhamento biométrico foram realizadas durante oito semanas: comprimento da folha, largura das folhas, altura da planta e número de folhas. Na época da coleta a diferença entre os vegetais plantados em diferentes substratos era facilmente perceptível conforme as Figuras 2, 3 e 4. Os vegetais plantados em terra vegetal cresceram mais rapidamente, conforme

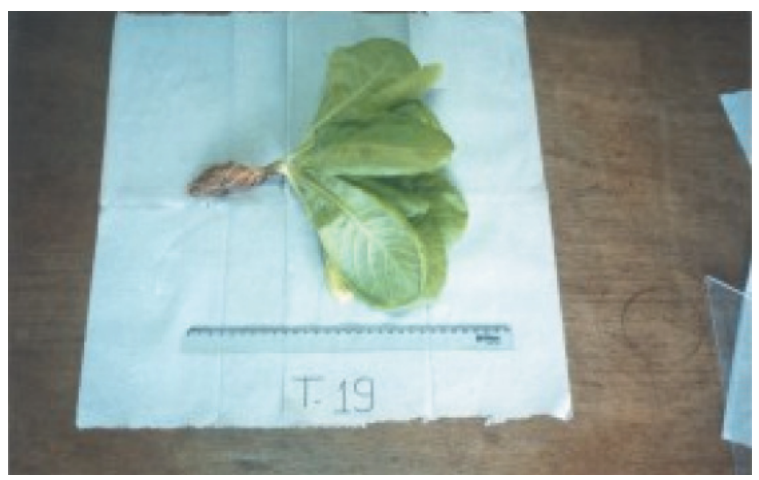

Figura 2. Alface plantada em terra vegetal

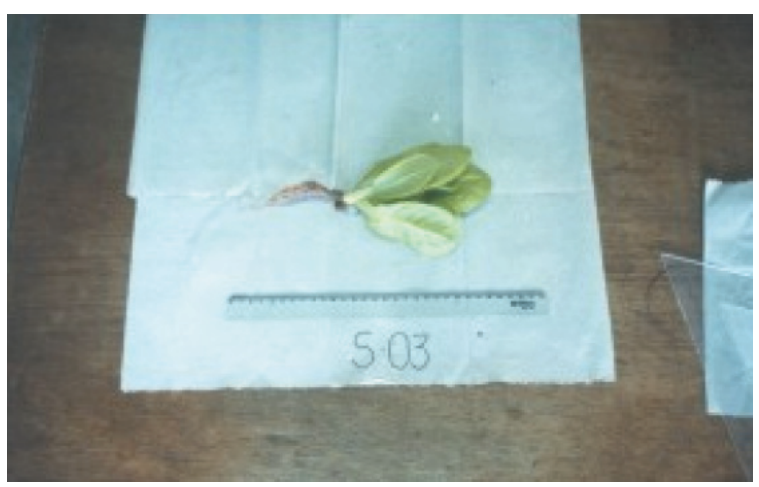

Figura 3. Alface plantada em mistura terra vegetal/sedimento

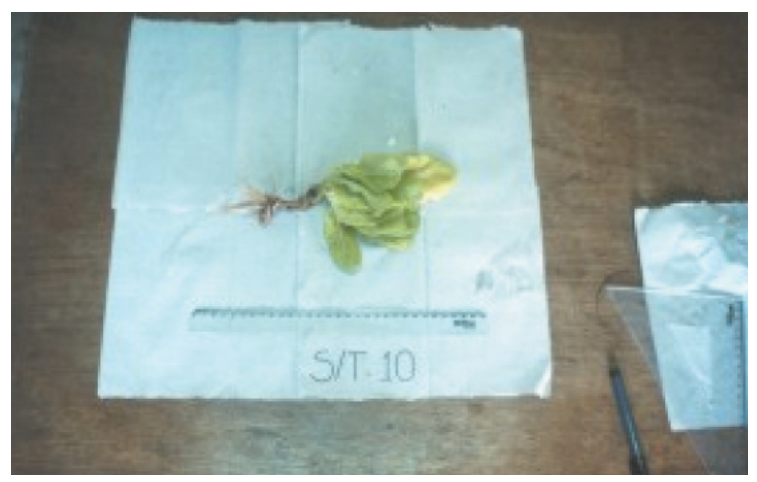

Figura 4. Alface plantada em sedimento 
vemos na Figura 5. A Figura 6 mostra a altura de uma folha média dos vegetais, para os diferentes substratos. A Figura 7 mostra o desenvolvimento da largura de uma folha média. Novamente nota-se que a terra vegetal produz melhores resultados. A mesma conclusão é válida para o número médio de folhas, mostrado na Figura 8.

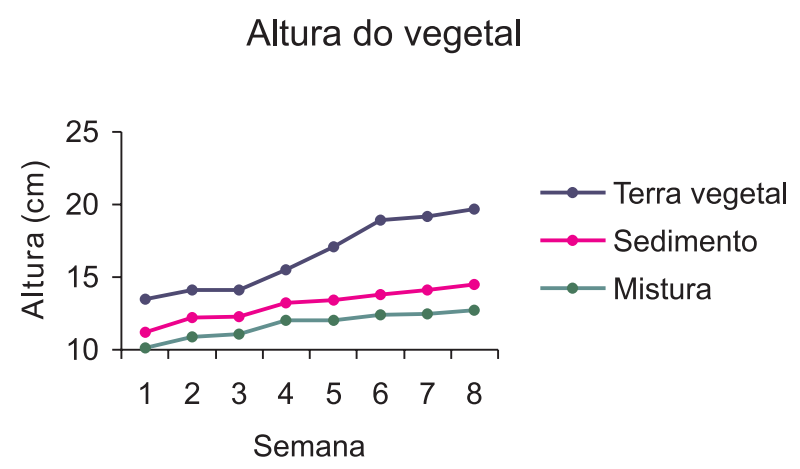

Figura 5. Medidas da altura do vegetal ao longo do tempo

\section{Altura da folha média}

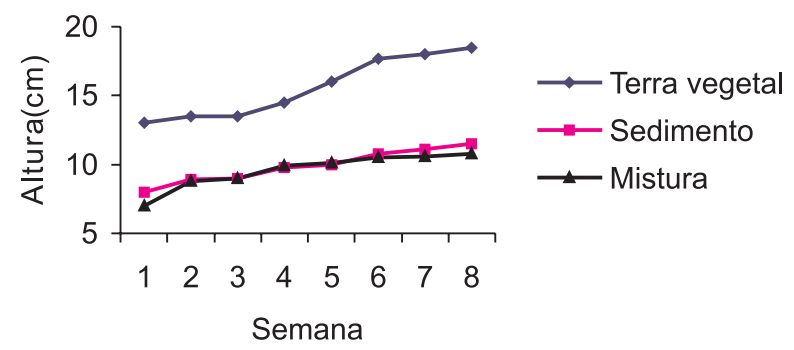

Figura 6. Medidas do crescimento de uma folha média ao longo do tempo

\section{Largura da folha média}

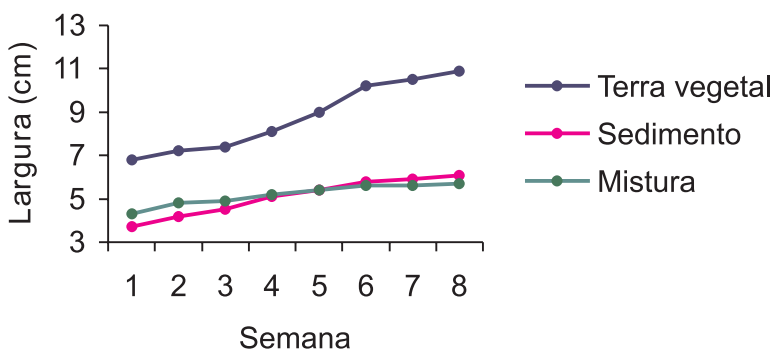

Figura 7. Medidas da largura de uma folha média ao longo do tempo

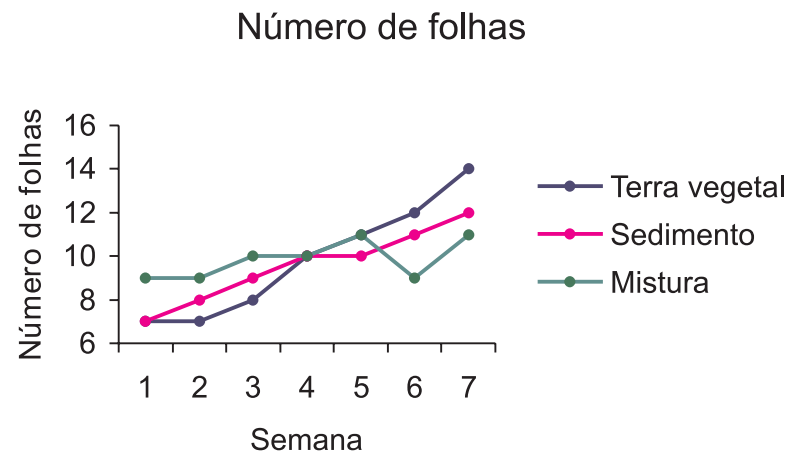

Figura 8. Medidas do número de folhas por vegetal ao longo do tempo

\section{Teor de umidade nas amostras}

A análise de variância aplicada ao fator de umidade demonstra que existe uma diferença significativa entre os três substratos. O resultado está apresentado na Figura 9.

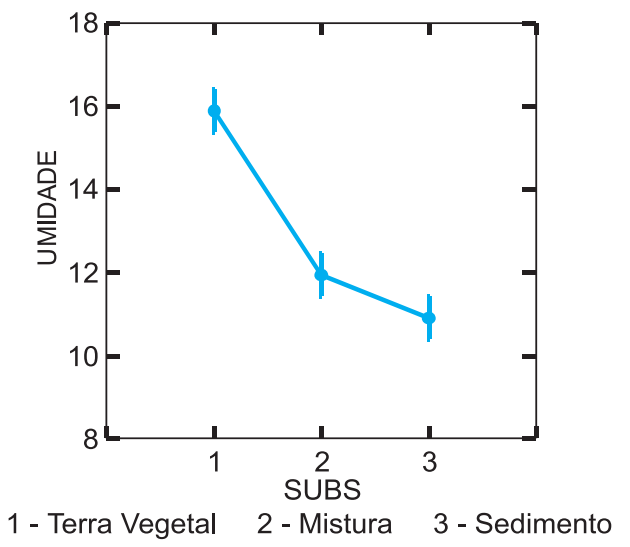

Figura 9. ANOVA aplicada ao fator de umidade das alfaces

\section{Teor de metais}

Os resultados, apresentados na Tabela 1 mostram que a concentração de As, Mn e Sc é maior na terra vegetal, enquanto que K e Na possuem valores menores. Já os demais elementos analisados apresentaram concentrações aproximadas para os dois substratos. A Tabela 1 apresenta também um quadro comparativo para as concentrações de elementos em alguns solos e sedimentos.

Os resultados mostraram que $\mathrm{Fe}, \mathrm{Co}, \mathrm{Cr}, \mathrm{Rb}$, e $\mathrm{Sc}$ apresentam maior concentração nas alfaces plantadas no sedimento da Lagoa da Pampulha e que Al, K, Mg, Na, e Zn apresentam maior a concentração nas plantadas em substrato com presença de terra vegetal. A Tabela 2 mostra os resultados obtidos para os vegetais plantados nos diferentes substratos, os valores certificados para o material de refe-

Tabela 1. Quadro comparativo da concentração dos elementos em solos e sedimentos

\begin{tabular}{cccccc}
\hline Elemento & $\begin{array}{c}\text { Solo 7 } \\
(\mathrm{ppm})\end{array}$ & $\begin{array}{c}\text { GXR6 } \\
(\mathrm{ppm})\end{array}$ & $\begin{array}{c}\text { Solo } \\
\text { poluído } \\
\text { de uma } \\
\text { fazenda } \\
(\mathrm{ppm})\end{array}$ & $\begin{array}{c}\text { Terra } \\
\text { vegetal } \\
(\mathrm{ppm})\end{array}$ & $\begin{array}{c}\text { Sedimento } \\
\text { da Lagoa } \\
\text { da } \\
\text { Pampulha } \\
(\mathrm{ppm})\end{array}$ \\
\hline $\mathrm{Al}$ & 47000 & 177000 & 68612 & 104000 & 113000 \\
$\mathrm{As}$ & 13,4 & 330 & 10,6 & 61 & $<10$ \\
$\mathrm{Ba}$ & 159 & 1300 & 72,4 & $<50$ & $<50$ \\
$\mathrm{Ce}$ & 61 & 36 & $*$ & $<50$ & $<50$ \\
$\mathrm{Co}$ & 8,9 & 13,8 & 13 & 4,6 & 4,1 \\
$\mathrm{Cr}$ & 60 & 96 & 112 & 40 & 38 \\
$\mathrm{Fe}$ & 25700 & 55800 & 29700 & 22400 & 18500 \\
$\mathrm{~K}$ & 12100 & 18700 & 15700 & 6600 & 13000 \\
$\mathrm{La}$ & 28 & 13,9 & 40 & 54 & 64 \\
$\mathrm{Mn}$ & 631 & 2400 & 519 & 510 & 360 \\
$\mathrm{Na}$ & 2400 & 1060 & 11000 & 370 & 900 \\
$\mathrm{Rb}$ & 51 & 90 & 68 & $<50$ & $<50$ \\
$\mathrm{Sc}$ & 8,3 & 27,6 & 10 & 7,5 & 5,2 \\
$\mathrm{Zn}$ & 104 & 118 & 260 & $<100$ & $<100$ \\
\hline
\end{tabular}

.Valor não informado 
Tabela 2. Quadro comparativo da concentração dos elementos em alface

\begin{tabular}{|c|c|c|c|c|c|c|c|c|c|c|c|}
\hline Planta & $\begin{array}{c}\mathrm{Al} \\
(\mu \mathrm{g} / \mathrm{g})\end{array}$ & $\begin{array}{c}\text { Co } \\
(\mu \mathrm{g} / \mathrm{g})\end{array}$ & $\begin{array}{c}\mathrm{Cr} \\
(\mu \mathrm{g} / \mathrm{g})\end{array}$ & $\begin{array}{c}\mathrm{Fe} \\
(\mu \mathrm{g} / \mathrm{g})\end{array}$ & $\begin{array}{c}\mathrm{K} \\
(\mu \mathrm{g} / \mathrm{g})\end{array}$ & $\begin{array}{c}\mathrm{Mg} \\
(\mu \mathrm{g} / \mathrm{g})\end{array}$ & $\begin{array}{c}\mathrm{Mn} \\
(\mu \mathrm{g} / \mathrm{g})\end{array}$ & $\begin{array}{c}\mathrm{Na} \\
(\mu \mathrm{g} / \mathrm{g})\end{array}$ & $\begin{array}{c}\mathrm{Rb} \\
(\mu \mathrm{g} / \mathrm{g})\end{array}$ & $\begin{array}{c}\mathrm{Sc} \\
(\mu \mathrm{g} / \mathrm{g})\end{array}$ & $\begin{array}{c}\mathrm{Zn} \\
(\mu \mathrm{g} / \mathrm{g})\end{array}$ \\
\hline Terra Vegetal & 50 & 0,0066 & 0,055 & 35 & 6450 & 520 & 9,2 & 166 & 10,6 & 0,006 & 7,7 \\
\hline Sedimento & 30 & 0,016 & 0,15 & 49 & 5140 & 210 & 8,6 & 56 & 12 & 0,011 & 4,7 \\
\hline $\begin{array}{l}\text { Mistura } \\
\text { (terra/sedimento) }\end{array}$ & 59 & 0,006 & 0,078 & 42 & 5790 & 320 & 6,3 & 95 & 5,2 & 0,008 & 7 \\
\hline Mixed $\operatorname{diet}^{* * *}$ & 4,39 & 0,038 & 0,102 & 37 & 7900 & 650 & 8,12 & 3120 & & & 17 \\
\hline Alface lisa* & & & & & 2570 & 130 & & 50 & & & \\
\hline Romana crua* & & & & 10 & 2900 & 60 & 10 & 80 & & & \\
\hline Crespa, crua* & & & & 10 & 1580 & 90 & & 90 & & & \\
\hline Americana, crua* & & & & 10 & 2640 & 110 & 10 & 90 & & & \\
\hline IDR $* *$ & $*$ & 0,09 & 15 & 1400 & 132000 & 20000 & 150 & 46000 & $*$ & $*$ & 1000 \\
\hline
\end{tabular}

*Fonte: USDA Nutrient Database for Standard Reference, Release 14 (Julho 2001) (http://www.unifesp.br/dis/servicos/nutri/nutri.php?id=2411); ** ingestão diária mínima recomendada $\mu \mathrm{g} / \mathrm{d}$; *** material de referência

rência Mixed diet e valores normalmente encontrados em algumas espécies de alface.

É possível perceber que os valores estão abaixo dos encontrados no material Mixed Diet, entretanto acima dos citados para outras espécies de alface, para Fe, $\mathrm{K}$ e $\mathrm{Mg}$.

\section{Análise dos dados}

A análise dos dados referentes à concentração de minerais na alface foi feita utilizando a distribuição logarítmica para suavizar a concentração de diferentes elementos ${ }^{9-11}$.

Como era de se esperar, percebe-se que o fator de umidade é negativamente correlacionado à concentração dos minerais, exceto $\mathrm{Cr}$, que apresentou baixa correlação $(0,406)$. A Figura 10 apresenta a distribuição do fator de umidade, em relação ao $\mathrm{Al}$ e $\mathrm{Na}$, na alface. Pode ser percebido que a umidade apresenta maior valor na alface plantada em terra vegetal enquanto os minerais apresentam maior valor na plantada em sedimento.
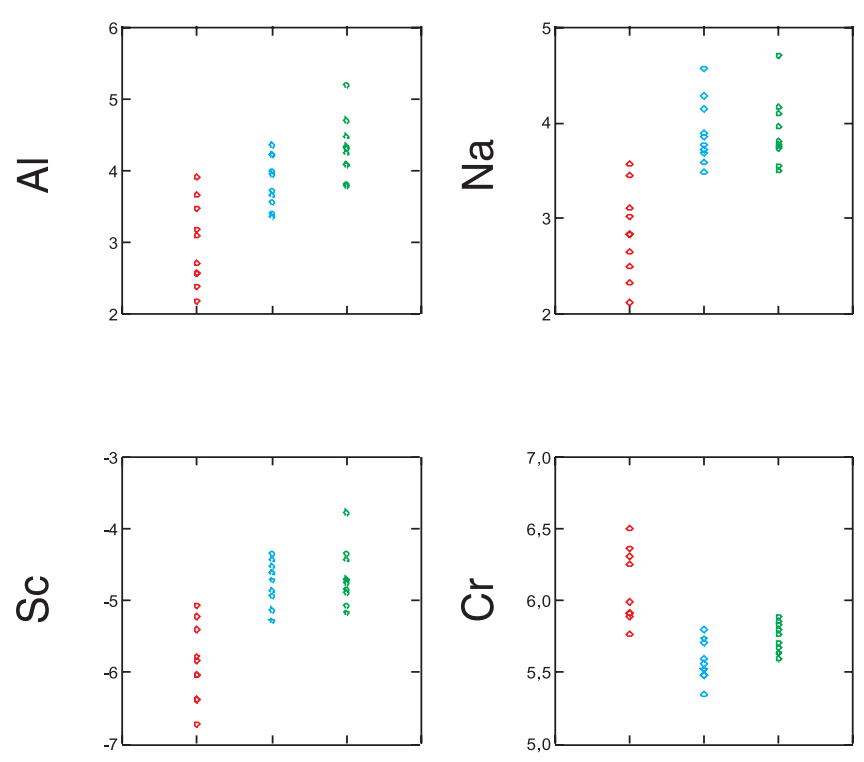

Mistura Sedimento

Terra vegetal
A redução de dados pela técnica de componentes principais mostra que 3 fatores representam $80 \%$ dos dados. O primeiro fator é caracterizado pela contribuição negativa da umidade e positiva do sódio e alumínio; o segundo, pela positiva do cromo e negativa do rubídio e o terceiro, pela positiva do zinco. A Figura 11 apresenta os fatores plotados. É perceptível que há diferença significativa entre os substratos. A análise de variância aplicada aos fatores demonstra que esta diferença é significativa. Os resultados estão apresentados na Figura 12.

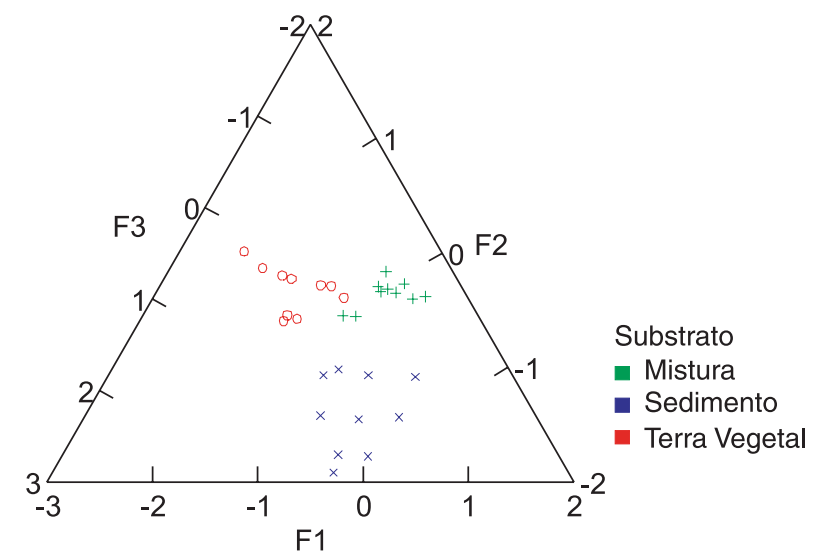

Figura 11. Distribuição dos fatores encontrados pela técnica de componentes principais (alface)

\section{CONCLUSÕES}

Os estudos efetuados demonstraram que o fator de umidade da alface cultivada em sedimentos da Lagoa da Pampulha é menor que o da cultivada em terra vegetal. O desenvolvimento no sedimento também é bastante inferior. Isto é um indício que a população provavelmente não utilizará o local de descarte destes sedimentos para plantio de alface, e talvez nem de hortigranjeiros.

A análise de diferentes solos mostrou que os valores encontrados tanto para a terra vegetal quanto para os sedimentos são menores ou iguais aos encontrados em outros solos e sedimentos, inclusive os utilizados internacionalmente como materiais de referência, o que permite afirmar que os sedimentos coletados não apresentam um nível significativo de contaminação.

É importante lembrar, entretanto, que a umidade da alface é menor, o que implica em maior densidade e, consequientemente, maior 

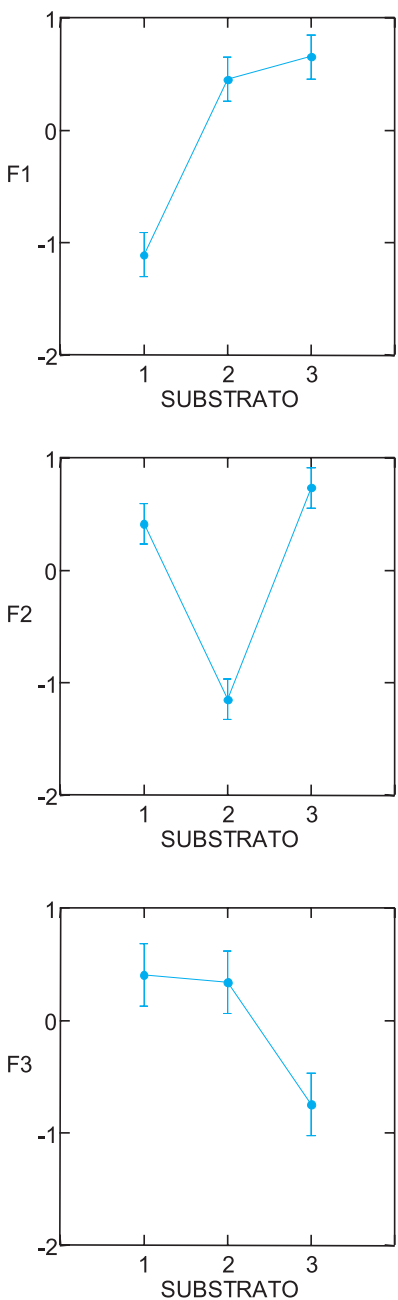

1-Terra Vegetal 2-Mistura 3-Sedimento

Figura 12. Análise da variância aplicada aos fatores (alface) concentração de minerais. Comparando-se os valores encontrados para a alface com a IDR percebe-se que, exceto para o Co, a ingestão média de alface por dia, que é $50 \mathrm{~g}$, não representaria risco.

Tendo em vista estes resultados, conclui-se que a remoção dos sedimentos depositados na Lagoa da Pampulha e seu despejo em áreas de bota-fora a jusante da represa, não implicará necessariamente em risco para a população local no que se refere à biodisponibilidade de metais nestes sedimentos.

\section{AGRADECIMENTOS}

Este projeto foi financiado pelo FIP (Fundo de Incentivo à Pesquisa) da Pontifícia Universidade Católica (PUC-Minas), e teve o apoio do Centro de Desenvolvimento da Tecnologia Nuclear (CDTN). Agradecemos também ao Curso de Mestrado em Ciências e Técnicas Nucleares da Escola de Engenharia da UFMG o apoio à realização deste trabalho, tema de dissertação de mestrado.

\section{REFERÊNCIAS}

1. Aun, P. E.; Bandeira, J.V. Em IAEA TECDOC 818: Use of nuclear techniques in studying soil erosion and siltation -Vienna, Austria, 1995.

2. Fioravante Neto, A.; Pinto, G. G.; Machado, M. L.; Vieira, N. S.; Bomtempo, V. L.; CDTN/CNEN, RC CT7003/95, 1995.

3. Bandeira, J. V.; Pinto, G. G.; Sabino, C. S.; Agudo, E. G.; Simpósio Brasileiro de Recursos Hídricos, Belo Horizonte, Brasil, 1999.

4. Superintendência de Desenvolvimento da Capital, SUDECAP, Março, 1996.

5. Soon-Oh, K.; Kyoung-Woong, K.; Stüben, D. J.; Environ. Eng. 2002, August, 705.

6. Santos, I. C.; Casali, V. W. D.; Miranda, G. V.; Pesquisa Agropecuária Brasileira 1998, 33, 157.

7. http://www.sementesfeltrin.com.br. acessada em Janeiro 2002.

8. De Corte, F.; Tese de Doutorado, Faculteit Van de Wetenschappen, Bélgica, 1986.

9. Bishop, R. L.; Neff, H.; Meeting of the American Chemical Society, Denver, USA, 1987.

10. Cheng, T. P.; Morris, J. S.; Koirtyohann, S. R.; Spate, V. L.; Baskett, C. K.; J. Radioanal. Nucl. Chem. 1995, 195, 31.

11. Shumilin E.; J. Radioanal. Nucl. Chem. 2000, 246, 533. 